\title{
PENERAPAN ALGORITMA MESSAGE DIGEST 5 PADA FILE VIDEO
}

\author{
Rivalri Kristianto Hondro \\ Universitas Budi Darma \\ Jalan Sisingamangaraja No. 338 Simpang Limun, Kota Medan \\ rivalryhondro@gmail.com
}

\begin{abstract}
Using the video document as a medium to convey information that is widely used today. Where proven in several social networking applications much content on the information in the form of video. Noting the security side of the video that is intended to secure the information in the videos cannot be accessed by unauthorized persons. In addition, the security side to ensure is rejected from the data that should be avoided-manipulation actions performed by people who are not responsible. Technical security video that can regulate the freedom or authenticity of the video cryptographic techniques. One algorithm cryptography was able to verify from the video is the message-digest algorithm 5. The results obtained from the application message digest 5 is a hash code that can be used as the value of the meter to the authenticity of the identity meta-approved video data.
\end{abstract}

Keyword: Authentication, Cryptography, Message Digest 5 Algorithm, Video

\section{PENDAHULUAN}

Pada jaman ini kebanyakan orang, instansi swasta dan pemerintahan menggunakan pengolahan data berbasis komputer sehingga menghasilkan representasi data dalam bentuk data digital. Bukan itu saja proses pendistribusian data juga dilakukan dengan menggunakan teknologi internet dengan memanfaatkan sejumlah aplikasi layanan pengiriman data seperti E-Mail dan aplikasi jejaringan social seperti Facebook, Twitter, Instagram, Whatsapp (Hondro, 2015). Maka dengan situasi seperti ini bisa menimbulkan beberapa tindakan penyadapan dan pemanipulasian data yang dilakukan oleh orang yang tidak berkepentingan, maka diperlukan sebuah otentikasi dokumen. Pada artikel
Hondro, dkk, menjelesakan bahwa Otentikasi merupakan kegiatan verifikasi dokumen digital dimana dokumen tersebut dapat diakses oleh orang yang berhak (Zebua, et al., 2018). Selain itu dapat juga memastikan dokumen digital itu asli atau tidak.

Video adalah salah satu jenis data digital yang digunakan orang saat ini untuk menyampaikan sebuah informasi. Informasi yang ada dalam video tentunya perlu dijaga keasliannya sehingga orang berkepentingan dapat meyakinkan dirinya bahwasannya video tersebut asli (Gunawan, 2018). Cara yang tepat untuk mengatasi permasalahan tersebut yaitu dengan menerapkan teknik kriptografi jenis hash (Purwanti et al., 2013). Dimana dengan menerpkan teknik ini 
maka dihasilkan sebuah kode identitas sebuah file (Hondro, et al., 2014). Algoritma kriptografi jenis hash yang dapat diterapkan dalam proses otentikasi file video yaitu algoritma message digest 5.

\section{METODE}

Algoritma message digest 5 adalah jenis fungsi hash satu arah. Fungsi hash adalah proses pemberian kode otentikasi terhadap file secara efisien yang mana akan mengubah string input dengan panjang tak terhingga menjadi string output dengan panjang tetap yang disebut nilai hash (Munir, 2019). Algoritma message digest 5 dibuat oleh seorang pakar kriptografi bernama Ronal Rivest pada tahun 1991(Waqidiyanto, 2018).



Gambar 1. Pembuatan message digest 5

Langkah-langkah pembuatan message digest 5 (Mogarala \& Kabadi, 2018):

1. Menambahkan padding bits.

a. Pesan ditambah dengan sejumlah padding bits sedemikian sehingga panjang pesan (dalam satuan bit) kongruen dengan 448 modulo 512.

b. Jika panjang pesan 448 bit, tambahkan 512-bit sehingga menjadi 960 bit. Jadi, panjang bit padding bits adalah antara 1 sampai 512.

c. Padding bits terdiri atas sebuah bit 1 dan, sisanya, yang mengikutinya, bit 0 .

2. Menambahkan nilai panjang pesan.

a. Pesan yang telah diberi padding bits selanjutnya ditambah lagi dengan 64-bit yang menyatakan panjang pesan semula.

b. Jika panjang pesan $>2^{64}$, yang diambil adalah panjangnya dalam modulo $2^{64}$. Dengan kata lain, jika panjang pesan semula adalah $\mathrm{K}$ bit, 64-bit yang ditambahkan menyatakan $\mathrm{K}$ modulo $2^{64}$.

c. Setelah ditambah dengan 64 bit, panjang pesan sekarang menjadi kelipatan 512 bit.

3. Menginisialisasi penyangga MD

a. MD5 membutuhkan 4 buah penyangga yang masing-masing panjangnya 32 bit. Total panjang penyangga adalah 4 x $32=128$ bit . Keempat penyangga ini menampung hasil antara dan hasil akhir.

b. Keempat penyangga dinamai $\mathrm{A}, \mathrm{B}$, C, dan D. Setiap penyangga 
dinisialisasi dengan nilai-nilai (dalam notasi $H E X$ ) berikut:

$\mathrm{A}=01234567$

$\mathrm{B}=89 \mathrm{AB} \mathrm{CD} \mathrm{EF}$

$\mathrm{C}=\mathrm{FE}$ DC BA 98

$\mathrm{D}=76543210$

4. Mengolah pesan dalam blok berukuran 512 bit.

a. Pesan dibagi menjadi L buah blok yang masing-masing panjangnya 512 bit $\left(Y_{0}\right.$ sampai $\left.Y_{L-1}\right)$.

b. Setiap blok 512-bit diproses bersama dengan penyangga MD menjadi keluaran 128-bit, yang disebut proses $H_{M D 5}$ seperti diperlihatkan pada Gambar 1 . Proses $H_{M D 5}$ terdiri atas 4 buah putaran. Masing-masing putaran melakukan operasi dasar memakai sebuah elemen T. Jadi, setiap putaran memakai 16 elemen tabel T.

c. Pada Gambar 1, Yq menyatakan blok 512-bit ke-q dari pesan yang telah ditambahi padding bits dan tambahan 64-bit nilai panjang pesan semula. MDq adalah nilai message digest 128-bit dari proses $H_{M D 5}$ ke-q. Pada awal proses, MDq berisi nilai inisialisasi penyangga MD.



Gambar 2. Pengolahan blok 512 bit (proses $\mathrm{H}_{S H A}$ ).

Fungsi-fungsi $f_{F}, f_{G}, f_{H}$, dan $f_{I}$ masing-masing berisi 16 kali operasi dasar terhadap masukan (Weis, S. A., Sarma, S. E., Rivest, R. L., \& Engels, 204 C.E.). Setiap operasi dasar menggunakan elemen tabel $T$. Operasi dasar MD5 dapat ditulis dalam bentuk persamaan pada gambar 2.1.

$\mathrm{a} \leftarrow \mathrm{b}+\operatorname{CLSs}(\mathrm{a}+\mathrm{g}(\mathrm{b}, \mathrm{c}, \mathrm{d})+\mathrm{X}[\mathrm{k}]+\mathrm{T}$ [i])

Keterangan:

a, b, c, d: empat buah peubah penyangga 32 bit (berisinilaipenyangga $\mathrm{A}, \mathrm{B}$, C, D)

g : salah satu fungsi F, G, H, I

CLSs : circular left shift sebanyak s bit

$\mathrm{X}[\mathrm{k}]$ : kelompok 32-bit ke-k dariblok 512-bit message ke-q.

Nilai k : 0 sampai 15

$\mathrm{T}$ [i] : elemen tabel $\mathrm{T}$ ke-I (32 bit) 


$$
+\quad: \begin{array}{ll}
\text { operasi } \\
\text { modulo } 232
\end{array}
$$

Fungsi $f_{F}, f_{G}, f_{H}$, dan $f_{I}$ adalah fungsi untuk memanipulasi masukan a, b, c, dan d dengan ukuran 32 byte Masing-masing fungsi dapat dilihat pada tabel 1

Tabel 1. Fungsi Fungsi dasar MD5

\begin{tabular}{|l|l|l|}
\hline Nama & Notasi & $\mathrm{G}(\mathrm{b}, \mathrm{c}, \mathrm{d})$ \\
\hline Ff & $\mathrm{F}(\mathrm{b}, \mathrm{c}, \mathrm{d})$ & $(\mathrm{b} \wedge \mathrm{c}) \vee(\sim \mathrm{b} \wedge \mathrm{d})$ \\
\hline Fg & $\mathrm{G}(\mathrm{b}, \mathrm{c}, \mathrm{d})$ & $(\mathrm{b} \wedge \mathrm{c}) \vee(\mathrm{c} \wedge \sim \mathrm{d})$ \\
\hline fH & $\mathrm{H}(\mathrm{b}, \mathrm{c}, \mathrm{d})$ & $\mathrm{b} \oplus \mathrm{c} \oplus \mathrm{d}$ \\
\hline Fi & $\mathrm{I}(\mathrm{b}, \mathrm{c}, \mathrm{d})$ & $\mathrm{c} \oplus(\mathrm{b} \wedge \sim \mathrm{d})$ \\
\hline
\end{tabular}

\begin{tabular}{|c|c|c|c|}
\hline $\begin{array}{l}\mathrm{T}[1]=\mathrm{D} 76 \mathrm{~A} \\
\mathrm{~A} 478\end{array}$ & $\begin{array}{l}\mathrm{T}[17]=\mathrm{F} 61 \mathrm{E} \\
2562\end{array}$ & $\begin{array}{l}\text { T[33]=FFFA } \\
3942\end{array}$ & $\begin{array}{l}\mathrm{T}[49]=F 429 \\
2244\end{array}$ \\
\hline $\begin{array}{l}\mathrm{T}[2]=\mathrm{E} 8 \mathrm{C} 7 \\
\mathrm{~B} 756\end{array}$ & $\begin{array}{l}\mathrm{T}[18]=\mathrm{C} 0 \mathrm{~B} 0 \\
\mathrm{~B} 340\end{array}$ & $\begin{array}{l}\mathrm{T}[34]=8771 \\
\mathrm{~F} 681\end{array}$ & $\begin{array}{l}\mathrm{T}[50]=432 \mathrm{~A} \\
\mathrm{FF} 97\end{array}$ \\
\hline $\begin{array}{l}\text { T3] }=242070 \\
\text { DB }\end{array}$ & $\begin{array}{l}\mathrm{T}[19]=265 \mathrm{E} \\
5 \mathrm{~A} 51\end{array}$ & $\begin{array}{l}T[35]=69 D 9 \\
6122\end{array}$ & $\begin{array}{l}\mathrm{T}[51]=\mathrm{AB} 94 \\
23 \mathrm{~A} 7\end{array}$ \\
\hline $\begin{array}{l}\mathrm{T}[4]=\mathrm{C} 1 \mathrm{BD} \\
\mathrm{CEEE}\end{array}$ & $\begin{array}{l}\text { T[20]=E9B6 } \\
\text { C7AA }\end{array}$ & $\begin{array}{l}\text { T[36]=FDE5 } \\
380 \mathrm{C}\end{array}$ & $\begin{array}{l}\text { T[52]=FC93 } \\
\text { A039 }\end{array}$ \\
\hline $\begin{array}{l}\text { T[5]=F57C0 } \\
\text { FAF }\end{array}$ & $\begin{array}{l}\mathrm{T}[21]=\mathrm{D} 62 \mathrm{~F} \\
105 \mathrm{D}\end{array}$ & $\begin{array}{l}\mathrm{T}[37]=A 4 B \\
\text { EEA44 }\end{array}$ & $\begin{array}{l}\mathrm{T}[53]= \\
655 \mathrm{~B} 59 \mathrm{C} 3\end{array}$ \\
\hline $\begin{array}{l}\mathrm{T}[6]=4787 \mathrm{C} \\
62 \mathrm{~A}\end{array}$ & $\begin{array}{l}\mathrm{T}[22]=0244 \\
1453\end{array}$ & $\begin{array}{l}\mathrm{T}[38]=4 \mathrm{BD} \\
\text { ECFA9 }\end{array}$ & $\begin{array}{l}\text { T[54]=8FOC } \\
\text { CC92 }\end{array}$ \\
\hline $\begin{array}{l}T[7]=A 8304 \\
613\end{array}$ & $\begin{array}{l}\mathrm{T}[23]=\mathrm{D} 8 \mathrm{~A} 1 \\
\mathrm{E} 681\end{array}$ & $\begin{array}{l}\text { T[39]=F6BB } \\
4 \mathrm{~B} 60\end{array}$ & $\begin{array}{l}\text { T[55]=FFEF } \\
\text { F47D }\end{array}$ \\
\hline $\begin{array}{l}\mathrm{T}[8]=\mathrm{FD} 469 \\
501\end{array}$ & $\begin{array}{l}\text { T[24]=E7D3 } \\
\text { FBCB }\end{array}$ & $\begin{array}{l}\mathrm{T}[40]=\mathrm{BEB} \\
\mathrm{FBC} 70\end{array}$ & $\begin{array}{l}\mathrm{T}[56]=85845 \\
\mathrm{DD} 1\end{array}$ \\
\hline $\begin{array}{l}\mathrm{T}[9]=69809 \\
8 \mathrm{D} 8\end{array}$ & $\begin{array}{l}\mathrm{T}[25]=21 \mathrm{E} 1 \\
\mathrm{CDE} 6\end{array}$ & $\begin{array}{l}\mathrm{T}[41]=289 \mathrm{~B} \\
7 \mathrm{EC} 6\end{array}$ & $\begin{array}{l}\text { T[57]=6FA8 } \\
7 \mathrm{E} 4 \mathrm{~F}\end{array}$ \\
\hline $\begin{array}{l}\text { T[10]=8B44 } \\
\text { F7AF }\end{array}$ & $\begin{array}{l}\mathrm{T}[26]=\mathrm{C} 337 \\
07 \mathrm{D} 6\end{array}$ & $\begin{array}{l}\mathrm{T}[42]=\mathrm{EAA} \\
127 \mathrm{FA}\end{array}$ & $\begin{array}{l}\text { T[58]=FE2C } \\
\text { E6E0 }\end{array}$ \\
\hline $\begin{array}{l}\text { T[11]=FFFF } \\
5 B B 1\end{array}$ & $\begin{array}{l}\text { T[27]=F4D5 } \\
\text { 0D87 }\end{array}$ & $\begin{array}{l}\text { T[43]=D4EF } \\
3085\end{array}$ & $\begin{array}{l}\mathrm{T}[59]=\mathrm{A} 301 \\
4314\end{array}$ \\
\hline $\begin{array}{l}\mathrm{T}[12]=895 \mathrm{C} \\
\mathrm{D} 7 \mathrm{BE}\end{array}$ & $\begin{array}{l}\mathrm{T}[28]=455 \mathrm{~A} \\
14 \mathrm{ED}\end{array}$ & $\begin{array}{l}\mathrm{T}[44]=0488 \\
\mathrm{D} 051\end{array}$ & $\begin{array}{l}\mathrm{T}] 60]=4 \mathrm{E} 08 \\
11 \mathrm{~A} 1\end{array}$ \\
\hline $\begin{array}{l}T[13]=6 B 90 \\
1122\end{array}$ & $\begin{array}{l}\text { T[29]=A9E3 } \\
\text { E905 }\end{array}$ & $\begin{array}{l}\text { T[45]=D9D4 } \\
\text { D039 }\end{array}$ & $\begin{array}{l}\mathrm{T}[61]=\mathrm{F} 753 \\
7 \mathrm{E} 82\end{array}$ \\
\hline $\begin{array}{l}\mathrm{T}[14]=\mathrm{FD} 98 \\
7193\end{array}$ & $\begin{array}{l}\text { T[30]=FCEF } \\
\text { A3F8 }\end{array}$ & $\begin{array}{l}\mathrm{T}[46]=\mathrm{E} 6 \mathrm{D} \\
\mathrm{B} 99 \mathrm{E} 5\end{array}$ & $\begin{array}{l}\mathrm{T}[62]=\mathrm{BD} 3 \\
\mathrm{AF} 235\end{array}$ \\
\hline $\begin{array}{l}T[15]=A 679 \\
438 E\end{array}$ & $\begin{array}{l}\mathrm{T}[31]=676 \mathrm{~F} \\
02 \mathrm{D} 9\end{array}$ & $\begin{array}{l}\mathrm{T}[47]=1 \mathrm{FA} 2 \\
7 \mathrm{CF} 8\end{array}$ & $\begin{array}{l}\mathrm{T}[63]=2 \mathrm{AD} 7 \\
\mathrm{D} 2 \mathrm{BB}\end{array}$ \\
\hline $\begin{array}{l}T[16]=49 B 4 \\
0821\end{array}$ & $\begin{array}{l}\mathrm{T}[32]=8 \mathrm{D} 2 \mathrm{~A} \\
4 \mathrm{C} 8 \mathrm{~A}\end{array}$ & $\begin{array}{l}\mathrm{T}[48]=\mathrm{C} 4 \mathrm{~A} \\
\mathrm{C} 5665\end{array}$ & $\begin{array}{l}\text { T[64]=EB86 } \\
\text { D391 }\end{array}$ \\
\hline
\end{tabular}

Tabel 2. Nilai T [1]

Dari Tabel 2 dapat dilihat bahwa masingmasing fungsi $f_{F}, f_{G}, f_{H}$, dan $f_{I}$ melakukan 16 operasi dasar .
Misalnya notasi [abcd k s i] menyatakan operasi.

$\mathrm{A} \leftarrow \mathrm{b}+((\mathrm{a}+\mathrm{g}((\mathrm{b}, \mathrm{c}, \mathrm{d})+\mathrm{X}[\mathrm{k}]+$ $\mathrm{T}[\mathrm{i}] \ll<<$ s)

Dimana $<<s$ s melambangkan dapat ditabulasikan sebagai berikut (Sadikin, 2012) :

1. Putaran 1:16 kali operasi dasar dengan $\mathrm{g}(\mathrm{b}, \mathrm{c}, \mathrm{d})=\mathrm{F}(\mathrm{b}, \mathrm{c}, \mathrm{d})$

Tabel 3. Perincian operasi pada fungsi

\begin{tabular}{|c|c|}
\hline No. & [abcd k s i] \\
\hline 1. & [ABCD $\left.0 \begin{array}{llll} & 7 & 1\end{array}\right]$ \\
\hline 2. & [DABC 11212 l \\
\hline 3. & [CDAB 2 17 3] \\
\hline 4. & [BCDA 3224$]$ \\
\hline 5. & [ABCD 47 5] \\
\hline 6. & [DABC 5 12 6] \\
\hline 7. & [CDAB 6 17 7] \\
\hline 8. & [BCDA 7228 ] \\
\hline 9. & [ABCD 879$]$ \\
\hline 10. & [DABC $9 \begin{array}{lll}\text { D } & 10]\end{array}$ \\
\hline 11. & [CDAB 1017 11] \\
\hline 12. & [BCDA 1122 12] \\
\hline 13. & [ABCD 127 13] \\
\hline 14. & {$\left[\begin{array}{lllll}\text { DABC } & 13 & 12 & 14\end{array}\right]$} \\
\hline 15. & [CDAB 14 17 15 ] \\
\hline 16. & [BCDA 1522 16] \\
\hline
\end{tabular}
$\mathrm{F}(\mathrm{b}, \mathrm{c}, \mathrm{d})$

2. Putaran 2:16 kali operasi dasar dengan $\mathrm{g}(\mathrm{b}, \mathrm{c}, \mathrm{d})=\mathrm{G}(\mathrm{b}, \mathrm{c}, \mathrm{d})$

\begin{tabular}{|c|c|}
\hline \multicolumn{2}{|r|}{$\mathrm{G}(\mathrm{b}, \mathrm{c}, \mathrm{d})$} \\
\hline No. & [abcd k s i] \\
\hline 17. & [ABCD 15 17] \\
\hline 18. & [DABC 699 18] \\
\hline 19. & [CDAB 1114 19] \\
\hline 20. & [BCDA 0 20 20] \\
\hline 21. & [ABCD 5 5 21] \\
\hline 22. & [DABC 10922$]$ \\
\hline 23. & [CDAB 15 14 14 23] \\
\hline 24. & [BCDA 420 24] \\
\hline 25. & [ABCD 95 5 25] \\
\hline
\end{tabular}

Tabel 4. Perincian operasi pada fungsi 


\begin{tabular}{|c|c|}
\hline 26. & [DABC 14926$]$ \\
\hline 27. & [CDAB 314 27] \\
\hline 28. & [BCDA 820 28] \\
\hline 29. & [ABCD 135 29] \\
\hline 30. & [DABC $\left.2 \begin{array}{llll} & 9 & 30\end{array}\right]$ \\
\hline 31. & [CDAB 714 31] \\
\hline 32. & [BCDA 12 20 32] \\
\hline
\end{tabular}

3. Putaran 3:16 kali operasi dasar dengan $\mathrm{g}(\mathrm{b}, \mathrm{c}, \mathrm{d})=\mathrm{H}(\mathrm{b}, \mathrm{c}, \mathrm{d})$

Tabel 5. Perincian operasi pada fungsi $\mathrm{H}(\mathrm{b}, \mathrm{c}, \mathrm{d})$

\begin{tabular}{|c|c|}
\hline No. & [abcd k s i] \\
\hline 33. & [ABCD 54 33] \\
\hline 34. & [DABC 8 llllll \\
\hline 35. & [CDAB 1116 16 \\
\hline 36. & [BCDA 1423 36] \\
\hline 37. & [ABCD 14 37] \\
\hline 38. & [DABC 4 llll 118 ] \\
\hline 39. & [CDAB 7 16 39] \\
\hline 40. & [BCDA 1023 40] \\
\hline 41. & [ABCD 13441$]$ \\
\hline 42. & [DABC $\left.\begin{array}{lllllllllll} & 0 & 11 & 42\end{array}\right]$ \\
\hline 43. & [CDAB 3 16 43] \\
\hline 44. & [BCDA 623 44] \\
\hline 45. & [ABCD 94 45] \\
\hline 46. & [DABC 121146$]$ \\
\hline 47. & [CDAB 1516 47] \\
\hline 48. & [BCDA 223 48] \\
\hline
\end{tabular}

4. Putaran 4:16 kali operasi dasar dengan $\mathrm{g}(\mathrm{b}, \mathrm{c}, \mathrm{d})=\mathrm{I}(\mathrm{b}, \mathrm{c}, \mathrm{d})$

Tabel 6. Perincian operasi pada fungsi $\mathrm{I}(\mathrm{b}, \mathrm{c}, \mathrm{d})$

\begin{tabular}{|c|c|}
\hline No & [abcd k s i] \\
\hline 49 & [ABCD $\left.0 \begin{array}{lll}0 & 49\end{array}\right]$ \\
\hline 50 & {$[\mathrm{DABC} 710$ 50] } \\
\hline 51 & [CDAB 141551 \\
\hline 52 & [BCDA 5 21 52] \\
\hline 53 & [ABCD 126 63] \\
\hline 54 & [DABC 310 (D4] \\
\hline 55 & [CDAB 101555 \\
\hline 56 & [BCDA 121 56] \\
\hline 57 & [ABCD 8657$]$ \\
\hline 58 & [DABC 151058 \\
\hline 59 & [CDAB 615 59] \\
\hline 60 & [BCDA 132160 \\
\hline 61 & [ABCD 46 61] \\
\hline
\end{tabular}

\begin{tabular}{|c|c|}
\hline 62 & [DABC 111062$]$ \\
\hline 63 & [CDAB 215 63] \\
\hline 64 & [BCDA 92164$]$ \\
\hline
\end{tabular}

Setelah putaran keempat, a, b, c, dan d ditambah kan ke A, B, C dan D. Selanjutnya, algoritma memproses blog data berikutnya $\left(\mathrm{Y}_{\mathrm{q}+1}\right)$. Keluaran akhir dari algoritma MD5 adalah hasil penyambungan bit di A, B, C, dan D. Dari uraian di atas, secara umum fungsi hash MD5 dapat ditulis dalam persamaan matematis berikut:

$\mathrm{MD}_{0}=\mathrm{IV}$

$\mathrm{MD}_{\mathrm{q}+1}=\mathrm{MDq}+\mathrm{fI}(\mathrm{Yq}+\mathrm{fH}(\mathrm{YQ}+$ $\mathrm{fF}(\mathrm{Yq}+\mathrm{MDq})))$

$$
\mathrm{MD}=\mathrm{MDL}-1
$$

Keterangan:

IV : initial vector dari penyangga $\mathrm{ABCD}$, yang dilakukan pada proses inisialisai penyangga

Yq : $\quad$ blok pesan berukuran 512bit ke-q

L : jumlah blok pesan

MD : $\quad$ nilai akhir message digest

\section{HASII DAN PEMBAHASAN}

1. Otentikasi File Video

Otentikasi file video dengan ekstensi file MP4 dilakukan dengan cara melakukan proses ekstrasi nilai terlebih dahulu, dengan menggunakan bantuan aplikasi Hex Workshop. Seperti pada tampilan berikut ini 


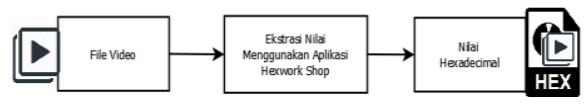

Gambar 3 Skema Ekstrasi Nilai Hexadecimal

Hasil proses penggunaan aplikasi hex workshop.

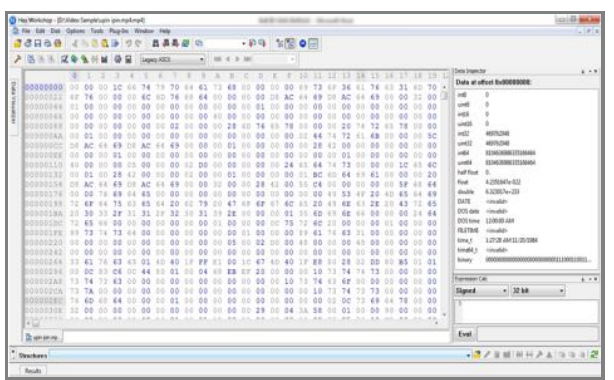

Gambar 4. Tampilan form Hex Workshop

2. Penerapan Algoritma Message Digest 5

Otentikasi terhadap file video

dilakukan dengan menerapkan algoritma message digest 5. Dari gambar 4 data video di atas diambil sebanyak 17-byte untuk sebagai sampel data penerapan MD5, yaitu: 000001C66747970646173680000000069

a. Menambah Padding Bits (Panjang Pesan)

Dari nilai hexadecimal sebagai sampel yang digunakan berjumlah 136-bit, agar pesan mencukupi 512-bit maka dilakukan penambahan bit, dimana bit pertama bernilai 1 (satu) selanjutnya diikuti bit 0 (nol). Delapan bit terakhir adalah nilai jumlah bit pesan yaitu $136=$ 10001000. Berikut hasil padding bit:

Tabel 7. Tabel Padding

\begin{tabular}{|l|l|l|l|l|l|l|}
\hline 00000000 & 00000000 & 00000000 & 00011100 & 01100110 & 01110100 & 01111001 \\
\hline 01110000 & 01100100 & 01100001 & 01110011 & 01101000 & 00000000 & 00000000 \\
\hline 00000000 & 00000000 & 01101001 & $\mathbf{1 0 0 0 0 0 0 0}$ & 00000000 & 00000000 & 00000000 \\
\hline 00000000 & 00000000 & 00000000 & 00000000 & 00000000 & 00000000 & 00000000 \\
\hline 00000000 & 00000000 & 00000000 & 00000000 & 00000000 & 00000000 & 00000000 \\
\hline 00000000 & 00000000 & 00000000 & 00000000 & 00000000 & 00000000 & 00000000 \\
\hline 00000000 & 00000000 & 00000000 & 00000000 & 00000000 & 00000000 & 00000000 \\
\hline 00000000 & 00000000 & 00000000 & 00000000 & 00000000 & 00000000 & 00000000 \\
\hline 00000000 & 00000000 & 00000000 & 00000000 & 00000000 & 00000000 & 00000000 \\
\hline 11111000 & \multicolumn{7}{|l|}{} \\
\hline
\end{tabular}


b. Inisialisasi Penyangga Nilai Hash Message Diggest (MD)

Fungsi $f_{F}, f_{G}, f_{H}$, dan $f_{I}$ adalah fungsi untuk memanipulasi masukan a, b, c, dan d dengan ukuran 32 bit, dan lihat table fungsi pada table 1

Penyelesaian fungsi fungsi dasar MD5:

1. $\mathrm{F}(\mathrm{b}, \mathrm{c}, \mathrm{d})=(\mathrm{b} \wedge \mathrm{c}) \vee(\sim \mathrm{b}$ $\wedge \mathrm{d})$

$=(89 \mathrm{ABCDEF} \wedge$ FEDCBA98) $\vee$ $(\sim 89 \mathrm{ABCDEF} \wedge$ 76543210)

a. $(b \wedge c)=(89 A B C D E F \wedge$ FEDCBA98)

b. $(\sim \mathrm{b} \wedge \mathrm{d})=(\sim 89 \mathrm{ABCDEF}$ $\wedge 76543210)$

c. $(\mathrm{b} \wedge \mathrm{c}) \vee(\sim \mathrm{b} \wedge \mathrm{d})$

Maka konversi nilai biner diatas ke Nilai Hexadecimal (Fungsi "F") = FEDCBA98. Lakukan hal sama dengan fungsi $\mathrm{G}, \mathrm{H}$, dan I.

2. $G(b, c, d)=(b \wedge d) \vee(c \wedge$ d) Hasil Nilai Hexadecimal (Fungsi “G”) $=88888888$
3. $\mathrm{H}(\mathrm{b}, \mathrm{c}, \mathrm{d})=\mathrm{b} \oplus \mathrm{c} \oplus \mathrm{d}$ Hasil Nilai Hexadecimal (Fungsi “H”) $=01234567$

4. $\quad \mathrm{I}(\mathrm{b}, \mathrm{c}, \mathrm{d})=\mathrm{c} \square(\mathrm{b} \wedge \sim \mathrm{d})$ Hasil Nilai Hexadecimal (Fungsi “I”) = 77777777

c. Pengolahan Pesan Dalam Blok Berukuran 512 Bit (Parsing) Bit pesan bagi menjadi 16 blok, dengan jumlah bit pada masingmasing blok berjumlah 32 bit

Selanjutnya lakukan perhitungan:

1. Putaran 1 - 16: menggunakan 16 kali operasi dasar dengan $\mathrm{g}(\mathrm{b}$, $\mathrm{c}, \mathrm{d})=\mathrm{F}(\mathrm{b}, \mathrm{c}, \mathrm{d})$

Putaran Ke - 1:

A $\square$ 89ABCDEF + $((01234567+$ FEDCBA98 + 0000001C + D76AA478) $<<<7)$

$01234567=00000001$ $\begin{array}{llll}0010 & 0011 & 0100 & 0101\end{array}$ 01100111

FEDCBA98 = 11111110 $\begin{array}{llll}1101 & 1100 & 1011 & 1010\end{array}$ 10011000

$\mathrm{M}_{0}=00000000 \quad 0000$ $\begin{array}{lllll}0000 & 0000 & 0000 & 0001\end{array}$ 1100 D76AA478 = 1101 $\begin{array}{llll}0111 & 0110 & 1010 & 1010\end{array}$ 010001111000 
Di XOR maka hasilnya

Hasil =11101 01110110 $\begin{array}{llll}1010 & 1010 & 0100 & 1001\end{array}$ 0011

Tulisan 1 bercetak tebal dibuang menjadi angka $1101 \quad 01110110 \quad 1010$ $\begin{array}{llll}1010 & 0100 & 1001 & 0011\end{array}$ $<<<7$

Tulisan bercetak tebal disebelah kiri digeser ke kanan

$\begin{array}{llll}1011 & 0101 \quad 0101 \quad 0010\end{array}$ 0100100111101011

89ABCDEF $=10001001$ $\begin{array}{llll}1010 & 1011 & 1100 & 1101\end{array}$ 11101111 Hasil $\mathrm{A}=$ $1 \underline{0011} \underline{1110} \quad \underline{1111} \underline{1110}$

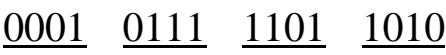
Nilai Hex $=3$ EFE17DA

Lakukan proses untuk 2 s/d 16 seperti proses putaran ke 1 dan ke 2, demikian juga dengan putaran ke $17 \mathrm{~s} / \mathrm{d} 32$, putaran $33 \mathrm{~s} / \mathrm{d}$ 48, dan putaran 49 s/d 64, dimana Putaran 17 - 32: menggunakan 16 kali operasi dasar dengan g (b, c, d) $=\mathrm{G}(\mathrm{b}, \mathrm{c}$, Putaran $33-48$ : menggunakan 16 kali operasi dasar dengan $\mathrm{g}(\mathrm{b}, \mathrm{c}, \mathrm{d})=\mathrm{H}(\mathrm{b}, \mathrm{c}$, Putaran 49 - 64: menggunakan 16 kali operasi dasar dengan g (b, c, $\mathrm{d})=\mathrm{I}(\mathrm{b}, \mathrm{c}$, Berikut hasil perputaran $1 \mathrm{~s} / \mathrm{d} 64$ pada tabel 8:

\begin{tabular}{|c|c|c|c|c|}
\hline Int & A & B & $\mathrm{C}$ & $\mathrm{D}$ \\
\hline$T_{a}$ & $\begin{array}{l}012345 \\
67\end{array}$ & $\begin{array}{l}\text { 89ABC } \\
\text { DEF }\end{array}$ & $\begin{array}{l}\text { FEDCB } \\
\text { A98 }\end{array}$ & $\begin{array}{l}765432 \\
10\end{array}$ \\
\hline$T_{1}$ & $\begin{array}{l}\text { 3EFE1 } \\
\text { 7DA }\end{array}$ & $\begin{array}{l}012345 \\
67\end{array}$ & $\begin{array}{l}89 \mathrm{ABC} \\
\text { DEF }\end{array}$ & $\begin{array}{l}\text { FEDC } \\
\text { BA98 }\end{array}$ \\
\hline$T_{\bar{L}}$ & $\begin{array}{l}\text { 4CB82 } \\
2 \mathrm{E} 2\end{array}$ & $\begin{array}{l}\text { 3EFE17 } \\
\text { DA }\end{array}$ & $\begin{array}{l}012345 \\
67\end{array}$ & $\begin{array}{l}\text { 89ABC } \\
\text { DEF }\end{array}$ \\
\hline$T_{\Xi}$ & $\begin{array}{l}\text { 5230D } \\
\text { EF2 }\end{array}$ & $\begin{array}{l}\text { 4CB82 } \\
2 \mathrm{E} 2\end{array}$ & $\begin{array}{l}\text { 3EFE17 } \\
\text { DA }\end{array}$ & $\begin{array}{l}012345 \\
67\end{array}$ \\
\hline$T_{4}$ & $\begin{array}{l}451 \mathrm{C} 3 \\
\mathrm{D} 62 \\
\end{array}$ & $\begin{array}{l}5230 \mathrm{D} \\
\mathrm{EF} 2\end{array}$ & $\begin{array}{l}4 \mathrm{CB} 82 \\
2 \mathrm{E} 2\end{array}$ & $\begin{array}{l}\text { 3EFE1 } \\
\text { 7DA }\end{array}$ \\
\hline$T_{9}$ & $\begin{array}{l}\text { 07B3A } \\
51 \mathrm{E}\end{array}$ & $\begin{array}{l}451 \mathrm{C} 3 \\
\text { D62 }\end{array}$ & $\begin{array}{l}\text { 5230D } \\
\text { EF2 }\end{array}$ & $\begin{array}{l}4 \mathrm{CB} 82 \\
2 \mathrm{E} 2\end{array}$ \\
\hline$T_{\text {t }}$ & $\begin{array}{l}\text { 064E5 } \\
267 \\
\end{array}$ & $\begin{array}{l}\text { 07B3A } \\
51 \mathrm{E}\end{array}$ & $\begin{array}{l}451 C 3 \\
\text { D62 }\end{array}$ & $\begin{array}{l}\text { 5230D } \\
\text { EF2 }\end{array}$ \\
\hline$T_{\bar{\tau}}$ & $\begin{array}{l}\text { 15D11 } \\
\text { E4F }\end{array}$ & $\begin{array}{l}\text { 064E52 } \\
67\end{array}$ & $\begin{array}{l}\text { 07B3A } \\
51 \mathrm{E}\end{array}$ & $\begin{array}{l}\text { 451C3 } \\
\text { D62 }\end{array}$ \\
\hline 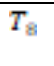 & $\begin{array}{l}\text { C9EB1 } \\
\text { F94 }\end{array}$ & $\begin{array}{l}\text { 15D11 } \\
\text { E4F }\end{array}$ & $\begin{array}{l}\text { 064E52 } \\
67\end{array}$ & $\begin{array}{l}\text { 07B3A } \\
51 \mathrm{E}\end{array}$ \\
\hline$T_{0}$ & $\begin{array}{l}\text { C9F83 } \\
9 \text { A3 }\end{array}$ & $\begin{array}{l}\text { C9EB1 } \\
\text { F94 }\end{array}$ & $\begin{array}{l}\text { 15D11 } \\
\text { E4F }\end{array}$ & $\begin{array}{l}\text { 064E5 } \\
267\end{array}$ \\
\hline$T_{10}$ & $\begin{array}{l}\text { D9263 } \\
\text { 6A3 }\end{array}$ & $\begin{array}{l}\text { C9F839 } \\
\text { A3 }\end{array}$ & $\begin{array}{l}\text { C9EB1 } \\
\text { F94 }\end{array}$ & $\begin{array}{l}\text { 15D11 } \\
\text { E4F }\end{array}$ \\
\hline$T_{\text {11 }}$ & $\begin{array}{l}7521 \mathrm{E} \\
\text { DEE }\end{array}$ & $\begin{array}{l}\text { D92636 } \\
\text { A3 }\end{array}$ & $\begin{array}{l}\text { C9F839 } \\
\text { A3 }\end{array}$ & $\begin{array}{l}\text { C9EB1 } \\
\text { F94 }\end{array}$ \\
\hline$T_{12}$ & $\begin{array}{l}790 \mathrm{E} 2 \\
524\end{array}$ & $\begin{array}{l}7521 \mathrm{E} \\
\text { DEE }\end{array}$ & $\begin{array}{l}\text { D92636 } \\
\text { A3 }\end{array}$ & $\begin{array}{l}\text { C9F83 } \\
9 \mathrm{~A} 3 \\
\end{array}$ \\
\hline$T_{19}$ & $\begin{array}{l}\text { 51B45 } \\
\text { EA4 }\end{array}$ & $\begin{array}{l}790 \mathrm{E} 25 \\
24\end{array}$ & $\begin{array}{l}7521 \mathrm{E} \\
\text { DEE }\end{array}$ & $\begin{array}{l}\text { D9263 } \\
\text { 6A3 }\end{array}$ \\
\hline$T_{14}$ & $\begin{array}{l}\text { 10C4F } \\
\text { DC8 }\end{array}$ & $\begin{array}{l}51 \mathrm{~B} 45 \mathrm{E} \\
\mathrm{A} 4\end{array}$ & $\begin{array}{l}790 \mathrm{E} 25 \\
24 \\
\end{array}$ & $\begin{array}{l}7521 \mathrm{E} \\
\text { DEE }\end{array}$ \\
\hline$T_{25}$ & $\begin{array}{l}10 \mathrm{C} 71 \\
\text { AE1 }\end{array}$ & $\begin{array}{l}\text { 10C4F } \\
\text { DC8 }\end{array}$ & $\begin{array}{l}51 \mathrm{~B} 45 \mathrm{E} \\
\mathrm{A} 4\end{array}$ & $\begin{array}{l}790 \mathrm{E} 2 \\
524\end{array}$ \\
\hline$T_{\text {II }}$ & $\begin{array}{l}\text { CFBE3 } \\
\text { AF1 }\end{array}$ & $\begin{array}{l}10 \mathrm{C} 71 \\
\text { AE1 }\end{array}$ & $\begin{array}{l}10 \mathrm{C} 4 \mathrm{~F} \\
\text { DC8 }\end{array}$ & $\begin{array}{l}\text { 51B45 } \\
\text { EA4 }\end{array}$ \\
\hline$T_{1 \%}$ & $\begin{array}{l}517966 \\
2 B\end{array}$ & $\begin{array}{l}\text { CFBE3 } \\
\text { AF1 }\end{array}$ & $\begin{array}{l}10 \mathrm{C} 71 \\
\text { AE1 }\end{array}$ & $\begin{array}{l}10 \mathrm{C} 4 \mathrm{~F} \\
\text { DC8 }\end{array}$ \\
\hline$T_{\text {II }}$ & $\begin{array}{l}\text { 62AE2 } \\
\text { C82 }\end{array}$ & $\begin{array}{l}517966 \\
2 B\end{array}$ & $\begin{array}{l}\text { CFBE3 } \\
\text { AF1 }\end{array}$ & $\begin{array}{l}10 \mathrm{C} 71 \\
\text { AE1 }\end{array}$ \\
\hline$T_{19}$ & $\begin{array}{l}13 \mathrm{BBF} \\
9 \mathrm{~F} 1\end{array}$ & $\begin{array}{l}\text { 62AE2 } \\
\text { C82 }\end{array}$ & $\begin{array}{l}517966 \\
2 B\end{array}$ & $\begin{array}{l}\text { CFBE3 } \\
\text { AF1 }\end{array}$ \\
\hline$T_{20}$ & $\begin{array}{l}\text { E5030 } \\
418\end{array}$ & $\begin{array}{l}\text { 13BBF } \\
9 \mathrm{~F} 1\end{array}$ & $\begin{array}{l}\text { 62AE2 } \\
\text { C82 }\end{array}$ & $\begin{array}{l}517966 \\
2 B\end{array}$ \\
\hline$T_{\text {21 }}$ & $\begin{array}{l}870797 \\
7 \mathrm{~A}\end{array}$ & $\begin{array}{l}\text { E50304 } \\
18\end{array}$ & $\begin{array}{l}\text { 13BBF } \\
9 \mathrm{~F} 1\end{array}$ & $\begin{array}{l}\text { 62AE2 } \\
\text { C82 }\end{array}$ \\
\hline$T_{2 z}$ & $\begin{array}{l}697053 \\
06\end{array}$ & $\begin{array}{l}870797 \\
7 \mathrm{~A}\end{array}$ & $\begin{array}{l}\text { E50304 } \\
18\end{array}$ & $\begin{array}{l}\text { 13BBF } \\
9 \mathrm{~F} 1\end{array}$ \\
\hline$T_{\text {¿ }}$ & $\begin{array}{l}\text { E705E } \\
682\end{array}$ & $\begin{array}{l}697053 \\
06\end{array}$ & $\begin{array}{l}870797 \\
7 \mathrm{~A}\end{array}$ & $\begin{array}{l}\text { E5030 } \\
418 \\
\end{array}$ \\
\hline$T_{24}$ & $\begin{array}{l}25597 \\
\text { DEB }\end{array}$ & $\begin{array}{l}\text { E705E6 } \\
82\end{array}$ & $\begin{array}{l}697053 \\
06\end{array}$ & $\begin{array}{l}870797 \\
7 \mathrm{~A}\end{array}$ \\
\hline$T_{\text {宝 }}$ & $\begin{array}{l}\text { FD5F4 } \\
\text { 8A4 }\end{array}$ & $\begin{array}{l}25597 \mathrm{D} \\
\mathrm{EB}\end{array}$ & $\begin{array}{l}\text { E705E6 } \\
82\end{array}$ & $\begin{array}{l}697053 \\
06\end{array}$ \\
\hline$T_{\mathrm{ZL}}$ & $\begin{array}{l}\text { 4F575 } \\
888\end{array}$ & $\begin{array}{l}\text { FD5F4 } \\
8 \text { A4 }\end{array}$ & $\begin{array}{l}25597 \mathrm{D} \\
\mathrm{EB}\end{array}$ & $\begin{array}{l}\text { E705E } \\
682\end{array}$ \\
\hline$T_{\text {TFF }}$ & $\begin{array}{l}\text { C2895 } \\
\text { D6B }\end{array}$ & $\begin{array}{l}\text { 4F5758 } \\
88\end{array}$ & $\begin{array}{l}\text { FD5F4 } \\
8 \mathrm{~A} 4\end{array}$ & $\begin{array}{l}25597 \\
\text { DEB }\end{array}$ \\
\hline$T_{\text {2: }}$ & $\begin{array}{l}\text { B178B } \\
\text { E4D }\end{array}$ & $\begin{array}{l}\text { C2895 } \\
\text { D6B }\end{array}$ & $\begin{array}{l}\text { 4F5758 } \\
88\end{array}$ & $\begin{array}{l}\text { FD5F4 } \\
\text { 8A4 }\end{array}$ \\
\hline$T_{20}$ & $\begin{array}{l}\text { FBA2 } \\
\text { AC75 }\end{array}$ & $\begin{array}{l}\text { B178B } \\
\text { E4D }\end{array}$ & $\begin{array}{l}\text { C2895 } \\
\text { D6B }\end{array}$ & $\begin{array}{l}\text { 4F5758 } \\
88\end{array}$ \\
\hline$T_{\mathrm{SO}}$ & $\begin{array}{l}83766 \\
\text { DC4 }\end{array}$ & $\begin{array}{l}\text { FBA2A } \\
\text { C75 }\end{array}$ & $\begin{array}{l}\text { B178B } \\
\text { E4D }\end{array}$ & $\begin{array}{l}\text { C2895 } \\
\text { D6B }\end{array}$ \\
\hline$T_{\text {TII }}$ & $\begin{array}{l}\text { 3DDE } \\
\text { 0A35 }\end{array}$ & $\begin{array}{l}83766 \mathrm{D} \\
\mathrm{C} 4\end{array}$ & $\begin{array}{l}\text { FBA2A } \\
\text { C75 }\end{array}$ & $\begin{array}{l}\text { B178B } \\
\text { E4D }\end{array}$ \\
\hline$T_{\Phi \underline{X I}}$ & $\begin{array}{l}\text { 313D3 } \\
\text { B50 }\end{array}$ & $\begin{array}{l}\text { 3DDE0 } \\
\text { A35 }\end{array}$ & $\begin{array}{l}83766 \mathrm{D} \\
\mathrm{C} 4\end{array}$ & $\begin{array}{l}\text { FBA2 } \\
\text { AC75 }\end{array}$ \\
\hline$T_{9 \mathrm{I}}$ & $\begin{array}{l}\text { ADB7 } \\
\text { EEEF }\end{array}$ & $\begin{array}{l}\text { 313D3 } \\
\text { B50 }\end{array}$ & $\begin{array}{l}\text { 3DDE0 } \\
\text { A35 }\end{array}$ & $\begin{array}{l}83766 \\
\text { DC4 } \\
\end{array}$ \\
\hline$T_{T_{4}}$ & $\begin{array}{l}\text { CDB6 } \\
4 \mathrm{~A} 3 \mathrm{C}\end{array}$ & $\begin{array}{l}\text { ADB7E } \\
\text { EEF }\end{array}$ & $\begin{array}{l}\text { 313D3 } \\
\text { B50 }\end{array}$ & $\begin{array}{l}\text { 3DDE0 } \\
\text { A35 }\end{array}$ \\
\hline
\end{tabular}




\begin{tabular}{|c|c|c|c|c|}
\hline$T_{\underline{y z}}$ & $\begin{array}{l}759 \mathrm{C} 3 \\
\text { A0E }\end{array}$ & $\begin{array}{l}\text { CDB64 } \\
\text { A3C }\end{array}$ & $\begin{array}{l}\text { ADB7E } \\
\text { EEF }\end{array}$ & $\begin{array}{l}\text { 313D3 } \\
\text { B50 }\end{array}$ \\
\hline$T_{95}$ & $\begin{array}{l}\text { F6AB } \\
\text { E3D0 }\end{array}$ & $\begin{array}{l}759 \mathrm{C} 3 \\
\text { A0E }\end{array}$ & $\begin{array}{l}\text { CDB64 } \\
\text { A3C }\end{array}$ & $\begin{array}{l}\text { ADB7 } \\
\text { EEEF }\end{array}$ \\
\hline$T_{9}$ & $\begin{array}{l}\text { 5D0A } \\
\text { B60F }\end{array}$ & $\begin{array}{l}\text { F6ABE } \\
\text { 3D0 }\end{array}$ & $\begin{array}{l}759 \mathrm{C} 3 \\
\text { A0E }\end{array}$ & $\begin{array}{l}\text { CDB64 } \\
\text { A3C }\end{array}$ \\
\hline$T_{99}$ & $\begin{array}{l}\text { B47F8 } \\
\text { BAC }\end{array}$ & $\begin{array}{l}\text { 5D0AB } \\
60 \mathrm{~F}\end{array}$ & $\begin{array}{l}\text { F6ABE } \\
\text { 3D0 }\end{array}$ & $\begin{array}{l}759 \mathrm{C} 3 \\
\mathrm{~A} 0 \mathrm{E}\end{array}$ \\
\hline$T_{90}$ & $\begin{array}{l}\text { 5FDA } \\
\text { C6F0 }\end{array}$ & $\begin{array}{l}\text { B47F8 } \\
\text { BAC }\end{array}$ & $\begin{array}{l}\text { 5D0AB } \\
60 \mathrm{~F}\end{array}$ & $\begin{array}{l}\text { F6ABE } \\
\text { 3D0 }\end{array}$ \\
\hline$T_{40}$ & $\begin{array}{l}290 \mathrm{C} 5 \\
112\end{array}$ & $\begin{array}{l}\text { 5FDAC } \\
6 \mathrm{~F} 0\end{array}$ & $\begin{array}{l}\text { B47F8 } \\
\text { BAC }\end{array}$ & $\begin{array}{l}\text { 5D0A } \\
\text { B60F }\end{array}$ \\
\hline$T_{41}$ & $\begin{array}{l}\text { 37CC6 } \\
731\end{array}$ & $\begin{array}{l}290 \mathrm{C} 51 \\
12\end{array}$ & $\begin{array}{l}\text { 5FDAC } \\
6 \mathrm{~F} 0\end{array}$ & $\begin{array}{l}\text { B47F8 } \\
\text { BAC }\end{array}$ \\
\hline$T_{4 \bar{L}}$ & $\begin{array}{l}\mathrm{C} 742 \mathrm{~F} \\
556\end{array}$ & $\begin{array}{l}37 \mathrm{CC} 6 \\
731 \\
\end{array}$ & $\begin{array}{l}290 \mathrm{C} 51 \\
12\end{array}$ & $\begin{array}{l}\text { 5FDA } \\
\text { C6F0 } \\
\end{array}$ \\
\hline$T_{42}$ & $\begin{array}{l}44 \mathrm{FFA} \\
524\end{array}$ & $\begin{array}{l}\text { C742F5 } \\
56 \\
\end{array}$ & $\begin{array}{l}37 \mathrm{CC} 6 \\
731\end{array}$ & $\begin{array}{l}290 \mathrm{C} 5 \\
112 \\
\end{array}$ \\
\hline$T_{44}$ & $\begin{array}{l}732 \mathrm{~F} 3 \\
542\end{array}$ & $\begin{array}{l}44 \mathrm{FFA} \\
524\end{array}$ & $\begin{array}{l}\text { C742F5 } \\
56\end{array}$ & $\begin{array}{l}37 \mathrm{CC} 6 \\
731\end{array}$ \\
\hline$T_{4}$ & $\begin{array}{l}\text { 4B617 } \\
\text { E6C }\end{array}$ & $\begin{array}{l}732 \mathrm{~F} 35 \\
42\end{array}$ & $\begin{array}{l}\text { 44FFA } \\
524\end{array}$ & $\begin{array}{l}\mathrm{C} 742 \mathrm{~F} \\
556\end{array}$ \\
\hline$T_{45}$ & $\begin{array}{l}\text { 9AD16 } \\
\text { D38 }\end{array}$ & $\begin{array}{l}\text { 4B617E } \\
6 \mathrm{C}\end{array}$ & $\begin{array}{l}732 \mathrm{~F} 35 \\
42\end{array}$ & $\begin{array}{l}\text { 44FFA } \\
524\end{array}$ \\
\hline$T_{4}$ & $\begin{array}{l}9269 \mathrm{E} \\
\text { FD8 }\end{array}$ & $\begin{array}{l}\text { 9AD16 } \\
\text { D38 }\end{array}$ & $\begin{array}{l}4 \mathrm{~B} 617 \mathrm{E} \\
6 \mathrm{C}\end{array}$ & $\begin{array}{l}732 \text { F35 } \\
42\end{array}$ \\
\hline$T_{48}$ & $\begin{array}{l}\text { D7417 } \\
819\end{array}$ & $\begin{array}{l}9269 \mathrm{EF} \\
\text { D8 }\end{array}$ & $\begin{array}{l}\text { 9AD16 } \\
\text { D38 }\end{array}$ & $\begin{array}{l}\text { 4B617 } \\
\text { E6C }\end{array}$ \\
\hline$T_{40}$ & $\begin{array}{l}\text { BAA3 } \\
9 D 8 A\end{array}$ & $\begin{array}{l}\text { D74178 } \\
19\end{array}$ & $\begin{array}{l}9269 \mathrm{EF} \\
\text { D8 }\end{array}$ & $\begin{array}{l}\text { 9AD16 } \\
\text { D38 }\end{array}$ \\
\hline$T_{90}$ & $\begin{array}{l}\text { A09D } \\
\text { A4DD }\end{array}$ & $\begin{array}{l}\text { BAA39 } \\
\text { D8A }\end{array}$ & $\begin{array}{l}\text { D74178 } \\
19\end{array}$ & $\begin{array}{l}9269 \mathrm{E} \\
\text { FD8 }\end{array}$ \\
\hline$T_{91}$ & $\begin{array}{l}\text { F9EE6 } \\
006\end{array}$ & $\begin{array}{l}\text { A09DA } \\
\text { 4DD }\end{array}$ & $\begin{array}{l}\text { BAA39 } \\
\text { D8A }\end{array}$ & $\begin{array}{l}\text { D7417 } \\
819 \\
\end{array}$ \\
\hline$\overline{s e x}$ & $\begin{array}{l}2 \mathrm{C} 9 \mathrm{~A} 7 \\
3 \mathrm{BA}\end{array}$ & $\begin{array}{l}\text { F9EE60 } \\
06\end{array}$ & $\begin{array}{l}\text { A09DA } \\
\text { 4DD }\end{array}$ & $\begin{array}{l}\text { BAA3 } \\
\text { 9D8A }\end{array}$ \\
\hline$T_{\mathrm{a}}$ & $\begin{array}{l}073175 \\
66\end{array}$ & $\begin{array}{l}\text { 2C9A7 } \\
3 \mathrm{BA}\end{array}$ & $\begin{array}{l}\text { F9EE60 } \\
06\end{array}$ & $\begin{array}{l}\text { A09D } \\
\text { A4DD }\end{array}$ \\
\hline$T_{Q_{4}}$ & $\begin{array}{l}\text { 27D18 } \\
\text { E0D }\end{array}$ & $\begin{array}{l}073175 \\
66 \\
\end{array}$ & $\begin{array}{l}2 \mathrm{C} 9 \mathrm{~A} 7 \\
3 \mathrm{BA}\end{array}$ & $\begin{array}{l}\text { F9EE6 } \\
006\end{array}$ \\
\hline$T_{T_{\text {SES }}}$ & $\begin{array}{l}\text { E2598 } \\
\text { A34 }\end{array}$ & $\begin{array}{l}\text { 27D18 } \\
\text { E0D }\end{array}$ & $\begin{array}{l}073175 \\
66\end{array}$ & $\begin{array}{l}\text { 2C9A7 } \\
3 \mathrm{BA}\end{array}$ \\
\hline$T_{\text {要 }}$ & $\begin{array}{l}\text { 0D986 } \\
061\end{array}$ & $\begin{array}{l}\text { E2598 } \\
\text { A34 }\end{array}$ & $\begin{array}{l}\text { 27D18 } \\
\text { E0D }\end{array}$ & $\begin{array}{l}073175 \\
66 \\
\end{array}$ \\
\hline$T_{9,8}$ & $\begin{array}{l}\text { 9A7A9 } \\
969\end{array}$ & $\begin{array}{l}\text { 0D9860 } \\
61\end{array}$ & $\begin{array}{l}\text { E2598 } \\
\text { A34 }\end{array}$ & $\begin{array}{l}\text { 27D18 } \\
\text { E0D }\end{array}$ \\
\hline $\bar{E}$ & $\begin{array}{l}\text { A83E } \\
\text { A7CA }\end{array}$ & $\begin{array}{l}\text { 9A7A9 } \\
969\end{array}$ & $\begin{array}{l}\text { 0D9860 } \\
61\end{array}$ & $\begin{array}{l}\text { E2598 } \\
\text { A34 }\end{array}$ \\
\hline$T_{90}$ & $\begin{array}{l}89 \mathrm{~A} 4 \mathrm{D} \\
\text { BBC }\end{array}$ & $\begin{array}{l}\text { A83EA } \\
7 \mathrm{CA}\end{array}$ & $\begin{array}{l}\text { 9A7A9 } \\
969\end{array}$ & $\begin{array}{l}\text { 0D986 } \\
061\end{array}$ \\
\hline 60 & $\begin{array}{l}59 \mathrm{~A} 4 \mathrm{~A} \\
248\end{array}$ & $\begin{array}{l}\text { 89A4D } \\
\text { BBC }\end{array}$ & $\begin{array}{l}\text { A83EA } \\
7 \mathrm{CA}\end{array}$ & $\begin{array}{l}\text { 9A7A9 } \\
969\end{array}$ \\
\hline$T_{181}$ & $\begin{array}{l}\text { 653AA } \\
5 \mathrm{~F} 4\end{array}$ & $\begin{array}{l}\text { 59A4A } \\
248 \\
\end{array}$ & $\begin{array}{l}\text { 89A4D } \\
\text { BBC }\end{array}$ & $\begin{array}{l}\text { A83EA } \\
7 \mathrm{CA}\end{array}$ \\
\hline$T_{\text {梳 }}$ & $\begin{array}{l}\text { E06BD } \\
\text { AC6 }\end{array}$ & $\begin{array}{l}\text { 653AA } \\
5 \mathrm{~F} 4\end{array}$ & $\begin{array}{l}59 \mathrm{~A} 4 \mathrm{~A} \\
248\end{array}$ & $\begin{array}{l}89 \mathrm{~A} 4 \mathrm{D} \\
\mathrm{BBC}\end{array}$ \\
\hline$T_{02}$ & $\begin{array}{l}8 \mathrm{AB} 45 \\
1 \mathrm{D} 9 \\
\end{array}$ & $\begin{array}{l}\text { E06BD } \\
\text { AC6 }\end{array}$ & $\begin{array}{l}\text { 653AA } \\
5 \mathrm{~F} 4\end{array}$ & $\begin{array}{l}59 \mathrm{~A} 4 \mathrm{~A} \\
248 \\
\end{array}$ \\
\hline
\end{tabular}

Setelah putaran ke 63 a,b,c, dan d ditambahkan ke A,B,C, dan D :

\begin{tabular}{|l|l|l|l|l|}
\hline $\mathbb{P}_{\text {[i] }}$ & $8 \mathrm{AB} 45$ & E06BD & $653 \mathrm{~A}$ & $59 \mathrm{~A} 4$ \\
& D 9 & AC6 & A5F4 & A248 \\
\hline A, B, & 012345 & $89 A B C$ & FEDC & 7654 \\
C, D & 67 & DEF & BA98 & 3210 \\
\hline Hasil & 899714 & $69 \mathrm{C} 01$ & 9BE61 & 2FF0 \\
& BE & 729 & F6C & 9058 \\
\hline
\end{tabular}

Penyelesaian:

a. 8AB451D9 $\oplus 01234567=$ 899714BE

b. E06BDAC6 $\oplus$ 89ABCDEF = 69C01729

c. $653 \mathrm{AA} 5 \mathrm{~F} 4 \oplus$ FEDCBA98 = 9BE61F6C

d. 59A4A248 $\oplus 76543210=$ 2FF09058

Jadi, hasil hash yang didapat dari file video Aku.mp4 adalah:

\section{BE69C017299BE61F6C}

\section{FF09058.}

Maka dengan kode hash tersebut dapat dijadikan sebagai identitas asli file video Aku.mp4.

\section{Hasil Pengujian}

Berikut hasil pengujian otentikasi file video dengan kasus perubahan yang berbeda-beda.

Tabel 9. Hasil Pengujian

\begin{tabular}{|c|c|c|c|c|c|}
\hline No & $\begin{array}{l}\text { Spesifi } \\
\text { kasi } \\
\text { File } \\
\text { Video } \\
\text { Asli }\end{array}$ & $\begin{array}{l}\text { Kode } \\
\text { Hash } \\
\text { File } \\
\text { Video } \\
\text { Asli }\end{array}$ & $\begin{array}{l}\text { Peru } \\
\text { baha } \\
\text { n } \\
\text { yang } \\
\text { dilak } \\
\text { ukan }\end{array}$ & $\begin{array}{l}\text { Spesif } \\
\text { ikasi } \\
\text { File } \\
\text { Video } \\
\text { Palsu }\end{array}$ & $\begin{array}{l}\text { Kode } \\
\text { Hash } \\
\text { File } \\
\text { Video } \\
\text { Asli }\end{array}$ \\
\hline 1 & $\begin{array}{l}\text { Nama } \\
\text { File: } \\
\text { Aku } \\
\text { Ukura } \\
\text { n: 1,3 } \\
\text { Mb } \\
\text { Durasi } \\
: \text { 10:00 } \\
\text { Jenis: } \\
\text { *.mp4 }\end{array}$ & $\begin{array}{l}89971 \\
\text { 4BE6 } \\
9 \mathrm{C} 017 \\
299 \mathrm{~B} \\
\text { E61F6 } \\
\text { C2FF } \\
09058\end{array}$ & $\begin{array}{l}\text { Mem } \\
\text { otong } \\
\text { durasi } \\
\text { video }\end{array}$ & $\begin{array}{l}\text { Nama } \\
\text { File: } \\
\text { Aku } \\
\text { Ukura } \\
\text { n: } 1 \\
\text { Mb } \\
\text { Durasi } \\
: \\
09: 55 \\
\text { Jenis: } \\
\text { *.mp4 }\end{array}$ & $\begin{array}{l}\text { A4E4 } \\
\text { 0F15 } \\
\text { AE54 } \\
2 \text { AE3 } \\
2 B 72 \\
34506 \\
11 \mathrm{~A} 5 \\
\text { CCB }\end{array}$ \\
\hline 2. & $\begin{array}{l}\text { Nama } \\
\text { File: } \\
\text { Video } \\
2 \\
\text { Ukura } \\
\mathrm{n}: \\
2 \mathrm{Mb} \\
\text { Durasi }\end{array}$ & $\begin{array}{l}0 \mathrm{C} 00 \\
\text { D49F } \\
80 \mathrm{EF} \\
\text { A785 } \\
\text { DDC } \\
\text { AD01 } \\
\text { A0FF } \\
\text { FF700 }\end{array}$ & $\begin{array}{l}\text { Mena } \\
\text { mbah } \\
\text { kontra } \\
\text { s } \\
\text { video }\end{array}$ & $\begin{array}{l}\text { Nama } \\
\text { File: } \\
\text { Video } \\
2 \\
\text { Ukura } \\
\mathrm{n}: \quad 2 \\
\mathrm{Mb} \\
\text { Durasi }\end{array}$ & $\begin{array}{l}\text { BA2D } \\
\text { DA48 } \\
\text { 1FDD } \\
\text { 08ED } \\
\text { DC8A } \\
\text { F0EA } \\
\text { 00A1 } \\
\text { FCA7 }\end{array}$ \\
\hline
\end{tabular}




\begin{tabular}{|c|c|c|c|c|c|}
\hline & $\begin{array}{l}: 12: 57 \\
\text { Jenis: } \\
\text { *.mp4 }\end{array}$ & & & $\begin{array}{l}: \\
\text { 13:01 } \\
\text { Jenis: } \\
\text { *.mp4 }\end{array}$ & \\
\hline \multirow[t]{10}{*}{3.} & Nama & DD0A & Mena & Nama & $0 \mathrm{C} 00$ \\
\hline & File: & C7F9 & mbah & File: & D49F \\
\hline & Sosial & COOE & water & Sosial & $80 \mathrm{EF}$ \\
\hline & Ukura & $1 \mathrm{AAF}$ & mark & Ukura & A785 \\
\hline & n: 820 & $5 \mathrm{CD} 4$ & didala & n: 998 & DDC \\
\hline & $\mathrm{Kb}$ & AE0D & & $\mathrm{Kb}$ & AD01 \\
\hline & Durasi & $\mathrm{AC} 0 \mathrm{~A}$ & video & Durasi & $\mathrm{A} 0 \mathrm{FF}$ \\
\hline & $: 01: 20$ & BB2E & & & EE70 \\
\hline & Jenis: & 0 & & $01: 20$ & 0 \\
\hline & *.mp4 & & & $\begin{array}{l}\text { Jenis: } \\
* \text { mp }\end{array}$ & \\
\hline
\end{tabular}

\section{KESIMPULAN}

Penerapan message digest 5 untuk otentikasi file video dapat menghasilkan kode hash yang dapat digunakan sebagai kode penanda identitas sebuah file video. Selain itu juga kode hash tersebut dapat dijadikan sebagai penanda apakah file video tersebut masih asli atau tidak.

\section{DAFTAR PUSTAKA}

Aumasson, J. P., Neves, S., WilcoxO'Hearn, Z., \& Winnerlein, C. (2013). BLAKE2: Simpler, smaller, fast as MD5. Lecture Notes in Computer Science (Including Subseries Lecture Notes in Artificial Intelligence and Lecture Notes in Bioinformatics). https://doi.org/10.1007/978-3-64238980-1_8

Gunawan, I. (2018). Penggunaan Algoritma Kriptografi Steganografi Least Significant Bit Untuk Pengamanan Pesan Teks dan Data Video. J-SAKTI (Jurnal Sains Komputer Dan Informatika). https://doi.org/10.30645/jsakti.v2i1.48

Hayati, N. (2017). Implementasi Algoritma RC4A dan MD5 untuk
Menjamin Confidentiality dan Integrity pada File Teks. Jurnal \& Penelitian Teknik Informatika.

Hondro, R. K. (2015). Aplikasi Enkripsi Dan Dekripsi SMS Dengan Algoritma Zig Zag Cipher Pada Mobile Phone Berbasis Android. Pelita Informatika Budi Darma, 3(10), 122-127. https://osf.io/preprints/inarxiv/a2dw $\mathrm{n} /$ download

Hondro, R. K. (2020a). Analisis Algoritma CLEFIA 128 Bit Jenis Block Cipher Untuk Pengamanan Teks. 1(2), 35-38.

Hondro, R. K. (2020b). Modifikasi Platform Kunci Algoritma Playfair Untuk Meningkatkan Nilai Confusion Pada Ciphertext. 1(2), 76-82.

Hondro, R. K., \& Nurcahyo, G. W. (2014). ANALISIS DAN PERANCANGAN SISTEM YANG MENERAPKAN ALGORITMA TRIANGLE CHAIN CIPHER (TCC) UNTUK ENKRIPSI RECORD TABEL DATABASE. Jurnal Teknologi Informasi Dan Komputer, 3(2), 118-127.

Mogarala, A. G., \& Kabadi, M. G. S. (2018). Proficient hamming weight based RSA-MD5 security for data storage in multi cloud environment. International Journal of Intelligent Engineering and Systems. https://doi.org/10.22266/IJIES2018. 0430.24

Munir, R. (2019). Kriptografi. In 2.

Purwanti, K., Hamdani, \& Septiarini, A. (2013). Kriptografi Pada Video Menggunakan Metode Transposisi. Jurnal Informatika Mulawarman. 
Sadikin, R. (2012). Kriptografi Untuk Keamanan Jaringan (I). ANDI OFFSET.

Sibyan, H. (2017). IMPLEMENTASI

ENKRIPSI BASIS DATA

DENGAN ALGORITMA MD5

(MESSAGE DIGEST

ALGORITHM 5) DAN

VIGENERE CIPHER. Ppkm I.

Turner, S., \& Chen, L. (2011). Updated Security Considerations for the

MD5 Message-Digest and the HMAC-MD5 Algorithms. RFC.

Waqidiyanto, A. (2018). No Proteksi URL Dengan Algoritma Md5 Dan Base 64 Untuk Pengamanan Website. Doctoral Dissertation.

Weis, S. A., Sarma, S. E., Rivest, R. L., \& Engels, D. W. (204 C.E.). Security and privacy aspects of lowcost radio frequency identification systems. In Security in pervasive computing (Heidelberg (ed.)). Springer.

Zebua, T., Hondro, R. K., \& Ndruru, E. (2018). Message Security on Chat App based on Massey Omura Algorithm. IJISTECH (International Journal Of Information System \& Technology), 1(2), 16-23. https://doi.org/10.30645/ijistech.v1i 2.11

Zheng, X., \& Jin, J. (2012). Research for the application and safety of MD5 algorithm in password authentication. Proceedings - 2012 9th International Conference on Fuzzy Systems and Knowledge Discovery, $\quad$ FSKD 2012. https://doi.org/10.1109/FSKD.2012. 6234010 